\title{
Reprogramming Fibroblasts into Cardiomyocytes
}

\author{
Charles E. Murry, M.D., Ph.D. and William T. Pu, M.D. \\ Departments of Pathology, Bioengineering, and Medicine/Cardiology, Center for Cardiovascular \\ Biology, Institute for Stem Cell and Regenerative Medicine, University of Washington, Seattle \\ (C.E.M.); the Department of Cardiology, Children's Hospital Boston, Boston (W.T.P.); and \\ Harvard Stem Cell Institute, Cambridge, MA (W.T.P).
}

A deficiency of cardiomyocytes underlies most cases of heart failure, and scientists have long sought to repopulate the heart with new cardiomyocytes. Most attempts at remuscularization have been based on the transplantation of stem cells or their derivatives, and success has been modest to date. A recent study by Ieda and colleagues ${ }^{1}$ points to a new therapeutic strategy: reprogramming fibroblasts to become cardiomyocytes.

The paradigm of cellular reprogramming originated with the discovery, in 1987, that expression of the skeletal muscle gene, $M Y O D 1$, could induce multiple cell types to differentiate into skeletal muscle. ${ }^{2} M Y O D 1$ encodes a transcription factor that regulates RNA transcription from target genes. This finding fueled the hypothesis that the activation of master transcription factors drives the differentiation of specific cell types. Unfortunately, most transcription factors appear to regulate only a subgroup of the genes necessary for the differentiation of different cell types, and a vigorous search did not identify other master regulators of equivalent potency to MYOD1. Interest in reprogramming waned until Takahashi and Yamanaka ${ }^{3}$ made the landmark discovery in 2006 that differentiated fibroblasts could be transcriptionally reprogrammed into induced pluripotent stem cells. ${ }^{3}$ Although no single transcription factor that was tested produced such cells, a combination of 25 transcription factors that are normally present in embryonic stem cells reprogrammed fibroblasts to pluripotency. A careful winnowing identified four transcription factors that were required for this reprogramming. Impressively, entire mice can now be generated from induced pluripotent stem cells, proving their bona fide stem-cell status.

Buoyed by these results, Ieda and colleagues expressed in mouse cardiac fibroblasts 14 cardiac regulatory genes, most of which encode transcription factors. To accelerate the screening process, the fibroblasts were engineered to express green fluorescent protein (GFP) on cardiac differentiation. Transduction with all 14 factors induced GFP expression in about $1 \%$ of cells. The 14 factors were carefully winnowed to 3 transcription factors Mef2C, Gata4, and Tbx 5 - that were necessary and sufficient to activate GFP expression in approximately $20 \%$ of cells (Fig. 1). Fibroblasts from the tail tip also could be reprogrammed in this way, albeit less efficiently, which effectively ruled out contamination by mature cardiomyocytes or a cardiac progenitor cell. Maintenance of the inducedcardiomyocyte state did not require continued expression of the reprogramming factors. When the fibroblasts were transplanted into the heart 1 day after transduction (but before activation of GFP), some of the cells underwent cardiac differentiation in vivo as well.

Activation of a cardiac-restricted reporter gene, of course, is not the same as full cardiac differentiation. One must look for loss of the fibro-blast phenotype, expression of

Copyright () 2011 Massachusetts Medical Society. All rights reserved.

Disclosure forms provided by the authors are available with the full text of this article at NEJM.org. 
endogenous cardiac proteins, and most important, evidence that the cells function as cardiomyocytes. Using microarrays to simultaneously profile thousands of RNA transcripts, Ieda et al. found that the GFP-positive cells down-regulated fibroblast markers, such as collagens, and expressed many cardiac genes. A minority of GFP-positive cells expressed other cardiac sarcomere genes and contained organized sarcomeres, and a small fraction displayed complex cardiac phenotypes, such as spontaneous calcium transients and spontaneous beating activity (Fig. 1). Thus, a subgroup of reprogrammed cells had physiological properties of cardiomyocytes, but the majority of cells were only partially reprogrammed.

Reprogramming of fibroblasts to cardiomyocytes holds tremendous potential for the treatment of heart failure. In theory, fibroblasts from a skin-biopsy sample, for example, could be used to generate autologous cardiomyocytes for repairing a patient's failing heart. Even more exciting would be the in situ reprogramming of fibroblasts in the injured heart. Such an approach might redirect these cells from scar formation to remuscularization, thus circumventing difficulties with graft survival and integration. Indeed, infarct fibroblasts have already been reprogrammed to skeletal muscle in situ with the use of MYOD1 transfer, providing an important proof of concept for this approach. ${ }^{4}$

Much remains to be done before this work is ready for clinical translation. The findings must be reproduced by independent researchers. Only a small minority (about 1\%) of reprogrammed myocytes appeared to be bona fide beating cardiomyocytes, so the efficiency of generating functioning cardiomyocytes requires substantial improvement. We need reprogramming systems without integrating viruses, and we need to know if the induced cardiomyocyte state persists over the long term. Finally, we must show that these cells can integrate into the injured heart, beat synchronously with the host myocardium, and restore electrical and contractile function. In the face of many potential roadblocks, the ability to reprogram fibroblasts to cardiomyocytes could revolutionize the field of cardiac repair.

\section{References}

1. Ieda M, Fu JD, Delgado-Olguin P, et al. Direct reprogramming of fibroblasts into functional cardiomyocytes by defined factors. Cell. 2010; 142:375-86. [PubMed: 20691899]

2. Davis RL, Weintraub H, Lassar AB. Expression of a single transfected cDNA converts fibroblasts to myoblasts. Cell. 1987; 51:987-1000. [PubMed: 3690668]

3. Takahashi K, Yamanaka S. Induction of pluripotent stem cells from mouse embryonic and adult fibroblast cultures by defined factors. Cell. 2006; 126:663-76. [PubMed: 16904174]

4. Murry CE, Kay MA, Bartosek T, Hauschka SD, Schwartz SM. Muscle differentiation during repair of myocardial necrosis in rats via gene transfer with MyoD. J Clin Invest. 1996; 98:2209-17. [PubMed: 8941636] 


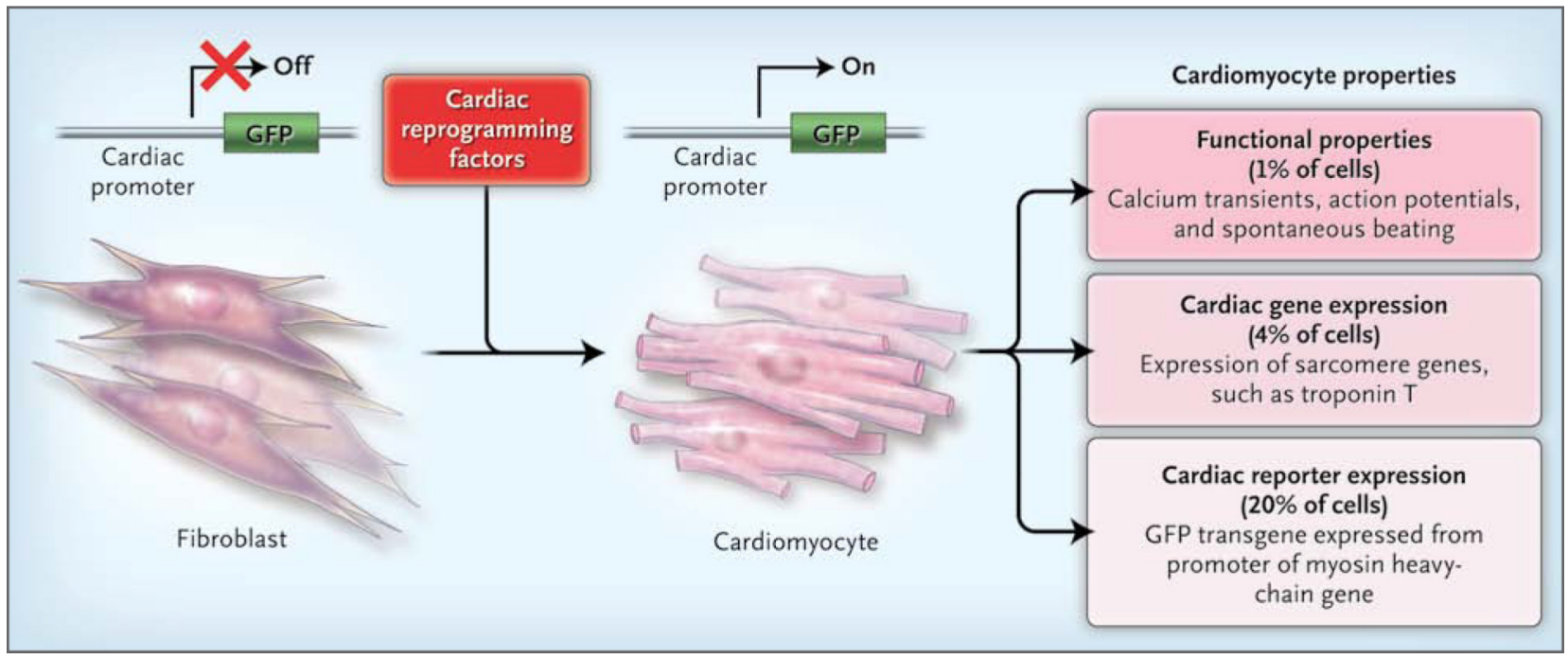

Figure 1. The Path from Fibroblast to Cardiomyocyte

Ieda and colleagues ${ }^{1}$ recently showed that the expression of specific transcription factors in the mouse fibroblast can instigate the cell's transdifferentiation into a cardiomyocyte, as evidenced by the expression of green fluorescent protein (GFP), which has been engineered in such a way that it is turned on only in cardiomyocytes. Reprogrammed cells attained different tiers of cardiomyocyte identity - for example, $20 \%$ had cardiac reporter expression through a GFP transgene, $4 \%$ had cardiac expression of sarcomere genes (e.g., troponin $\mathrm{T}$ ), and $1 \%$ showed spontaneous contractions and other functional properties of cardiomyocytes. 